\title{
COMPARISON OF THE MINISIM AND STISIM DRIVING SIMULATORS FOR THE DETECTION OF IMPAIRMENT: AN ALCOHOL VALIDATION STUDY
}

\author{
Gary Kay ${ }^{1}$, Omar Ahmad ${ }^{2}$, Timothy Brown ${ }^{2}$ \& Andrew Veit ${ }^{2}$ \\ ${ }^{1}$ Cognitive Research Corporation, Saint Petersburg, Florida, USA \\ ${ }^{2}$ University of Iowa, Iowa City, Iowa, USA \\ Email: gkay@cogres.com
}

\begin{abstract}
Summary: Detection of alcohol impairment is often used to evaluate the sensitivity of a protocol to detect the effects of other types of impairment. This study was designed to compare the sensitivity of two simulator platforms with different underlying architectures using equivalent driving scenarios. The driving scenario consisted of a twenty minute drive on a relatively straight rural roadway with a divided attention task presented infrequently during the drive. A total of 18 subjects completed drives on both simulators at two levels of BAC. It was hypothesized that both simulator platforms would be sensitive to the effects of alcohol. On driving variables and on divided attention variables the MiniSim simulator showed greater sensitivity to the impairing effects of alcohol (at doses below $0.10 \%$ BAC) than was found with the STI simulator. The SDLP variable (lane position deviation) was sensitive to alcohol effects with both simulators. However, there was clearly greater sensitivity seen with the MiniSim simulator. For a number of driving and divided attention variables significant results were obtained with the MiniSim, whereas results for the STISIM failed to show a significant alcohol effect. The greater sensitivity of the MiniSim compared to the STISIM is potentially due to a number of differences between the two simulators, though the difference in the vehicle dynamics model would be expected to be the largest determining factor.
\end{abstract}

\section{OBJECTIVE}

Prior to using PC-based driving simulators to evaluate drug effects it is first necessary to validate these simulators with respect to their sensitivity to detect a known impairing agent. STISIM is a well-established PC-based driving simulator which has been used in studies evaluating the impairing or enhancing effects of drugs (Kay \& Feldman, in press; Thompson, et al, 2010; Kay et al, 2009; Partinen, et al, 2003; Iudice, et al, 2002). The MiniSim driving simulator, an advanced, newer generation PC-based simulator, shows promise for use in clinical trials, but has not previously been validated with respect to its sensitivity to detect impairment. The present study provides a comparison of the sensitivity of these two driving simulators to detect alcohol. Alcohol is known to impair driving (simulated and actual driving) in a dose-related manner. This pilot study was designed to assess the relative sensitivity of the MiniSim and STISIM PCbased driving simulators for detecting central nervous system (CNS) impairment.

The primary objective of this study is to determine the relative sensitivity of two PC-based driving simulators (STISIM and MiniSim) to the effects of an impairing dose of alcohol (i.e., BAC levels $\geq 0.08 \%$. The desktop simulators (STISIM and MiniSim) will be sensitive to alcohol impairment observed as degradations in driving performance at blood alcohol 
concentrations (BAC level) $\geq 0.08 \%$ compared to performance to the placebo condition (BAC level $=0.00 \%)$.

\section{METHOD}

This study involved three visits: one screening visit and two dosing visits. Eighteen healthy subjects ages 21 to 34 years old were screened for study eligibility prior to the alcohol dosing visits. At the screening visit subjects provided a urine sample for a urine drug screen, vital signs were evaluated, a breath sample was obtained, and questionnaires were administered to determine alcohol use history. Female subjects provided a urine sample for pregnancy testing. Subjects completed a sample driving scenario to screen for simulator sickness. Additionally, subjects completed the questionnaires to assess the quantity-frequency-variability of their alcohol consumption (Cahalan, Cisin \& Crossley, 1969), the Alcohol Use Disorders Identification Test (Saunders et al, 1993) which determines if the subject is a chronic drinker, and the NADS Driving Survey to obtain demographic information, questions about their driving record, driving behaviors, driving history, health status, alcohol consumption history, and drinking behaviors. Subjects who meet screening criteria for entrance into the study were transported to and from the study center for visits 2 and 3. For visits 2 and 3, which occurred 2-7 days apart, subjects were again screened to determine if they continued to meet study entrance criteria.

Alcohol dosing was calculated based on the subject's drinking practices, height, and weight (Lee et al, 2010). The order in which subjects received alcohol and placebo was balanced. Subjects were dosed with alcohol to achieve a $0.10 \%$ BAC or received a placebo dose, $0.00 \%$ BAC. The drinks were a mixture of 1.5 parts orange juice to 1 part vodka or water based on subject characteristics. Additionally, for the placebo drink, the rim of the glass was swabbed with alcohol and a small quantity of alcohol was floated.

After arrival for the dosing visits and continued eligibility was confirmed, the subjects completed questionnaires about their sleep patterns within the last 24 hours and food intake within the last 4 hours, and were then dosed. Each subject received three drinks over a 30 minute period of time with a drink being served every 10 minutes. Sixteen minutes after the end of dosing, the first BAC measurement was taken and recorded. BAC measurements were taken until the subject had achieved the desired BAC $\pm 0.005 \%$ and was on the decline represented by successive measurements of declining BAC. Subjects then drove three simulator scenario drives: the first being on the MiniSim (using scenarios from a prior study conducted on full motion NADS-1 simulator (Lee et al, 2010) for comparison with the previously collected data), and then either MiniSim followed by STISIM or STISIM followed by MiniSim. Driving scenarios are described below. Only data from the second and third drive were analyzed for this comparison. Subjects completed questionnaires about current sleepiness levels before and after drives, how they felt after completing their drives, and how real they thought the simulators drove. Subjects were transported home once their BAC was below $0.003 \%$.

\section{Method of Randomization}

Subjects were randomly assigned to treatment order with half of the subjects receiving alcohol and half of the subjects receiving placebo at Visit 2. Subjects were crossed-over to the other 
treatment condition for Visit 3. Order of simulator driving was also randomized. After completing the first scenario on the MiniSim, subjects either drove the MiniSim followed by the STISIM; or drove the STISIM followed by the MiniSim for the two vigilance scenario drives.

\section{Driving Scenarios}

At Visits 2 \& 3, subjects completed three driving scenarios, only two of which are included in this analysis. The first drive completed was a drive that included urban, interstate and rural driving that was used in Lee et al (2010). After completing this scenario subjects drove two nearly identical Vigilance Scenarios which were designed to provide the same road geometry over the course of the simulated drive. The Vigilance Scenario is a 20-minute drive consisting of a 2-lane rural highway with no intersections or traffic control signals. The driver's tasks include maintaining speed at the specified speed limit (55 mph) while maintaining a stable lane position and also performing a secondary visual vigilance task (i.e., responding to low frequency targets by pressing a button on the steering wheel.

\section{Driving Simulators}

The two PC-based driving simulators, MiniSim and STISIM version 2 have been described in detail (Veit 2011; Kay etal, 2009). Both simulators used the same driving controls (ECCI Trackstar GT6000).

\section{Dependent Measures}

The primary dependent measure was standard deviation of lane position. The primary and secondary driving performance measures are summarized in Table 1. All driving performance measures were calculated over the course of the entire drive. Divided attention measures were also calculated and are summarized in Table 2.

Table 1. Driving Performance Measures

\begin{tabular}{|l|l|l|}
\hline Measure & Description & Units \\
\hline Standard Deviation of Lane Position & Steering control variability. & Feet \\
\hline Standard Deviation of Speed & Speed control variability. & Miles per hour \\
\hline Out of Lane & $\begin{array}{l}\text { Number of times that the vehicle crosses the lane } \\
\text { boundary. }\end{array}$ & Count \\
\hline Excessive Cornering Speed & $\begin{array}{l}\text { This score counts the number of times that the vehicle } \\
\text { exceeds a set g-level when cornering. }\end{array}$ & Count \\
\hline
\end{tabular}

Table 2. Divided Attention Measures

\begin{tabular}{|l|l|l|}
\hline Measure & Description & Units \\
\hline Correct Response & Correct responses to divided attention target. & Count \\
\hline Omission Errors & $\begin{array}{l}\text { Number of times subject fails to respond to divided } \\
\text { attention target. }\end{array}$ & Count \\
\hline Commission Errors & $\begin{array}{l}\text { Number of times subject responds to non-target stimuli } \\
\text { during divided attention task. }\end{array}$ & Count \\
\hline Reaction Time & Median reaction time for correct responses. & Seconds \\
\hline Accuracy & $\begin{array}{l}\text { Percent of correct responses relative to total responses } \\
\text { and omissions. }\end{array}$ & Percent \\
\hline
\end{tabular}




\section{RESULTS}

For comparison of the sensitivity of the two driving simulators results for the primary driving and divided attention variables generated by the Vigilance Scenario were compared by paired TTests. The observed Alcohol Levels for each of the treatment conditions and simulators are reported. Error bars in the plots represent standard error.

\section{Driving}

For Standard Deviation of Lane Position (SDLP) both simulators were found to be sensitive to the effects of alcohol, though the results suggest that the MiniSim may have greater sensitivity. For the STISIM the t-statistic of $-2.822(\mathrm{df}=17)$ yields a 2-tailed p-value of .012. By comparison for MiniSim the t-statistic of $-5.089(\mathrm{df}=17)$ yields a 2 -tailed $\mathrm{p}$-value of $<.001$. The relationships of the means for each condition are shown in Figure 1. As can be seen, SLDP scores are higher for the MiniSim. Comparison of the mean SDLP scores under the alcohol condition shows the STISIM mean at 1.32 with a Standard Error of .09 (6.8\% of the mean). By comparison, for the MiniSim the mean score is 2.25 and the Standard Error is 0.12 (5.3\% of the mean).

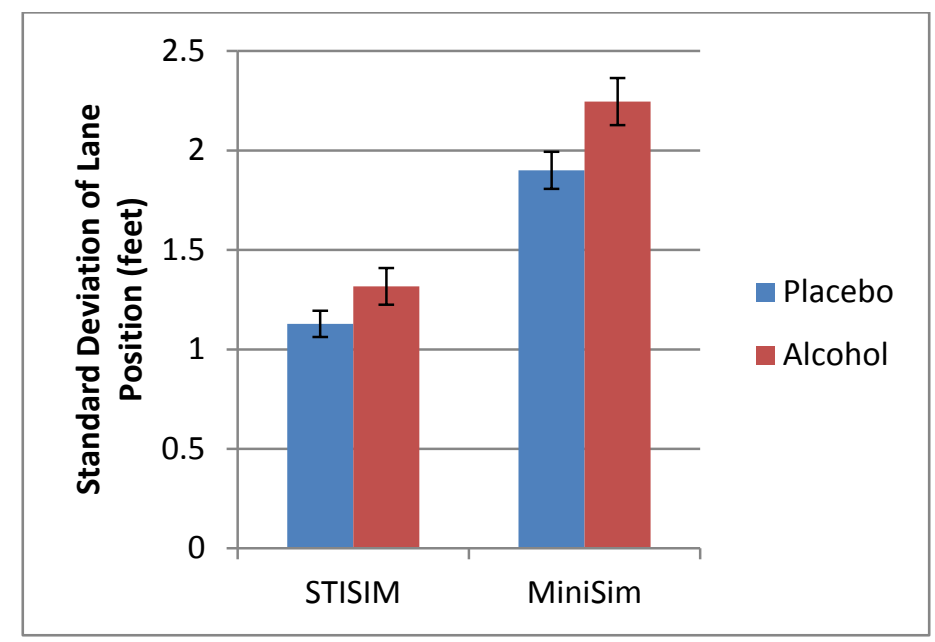

Figure 1. Variability in Lane Keeping

Typically, the second most important driving variable in impairment studies is standard deviation of speed. Results for the two simulators are shown in Figure 2. There was greater speed variability when driving the STISIM simulator under both treatment conditions. The STISIM simulator failed to detect a difference between alcohol and placebo for speed deviation $(\mathrm{t}=-.29$; $\mathrm{df}=17 ; \mathrm{p}=.775)$. By contrast for the MiniSim the speed deviation difference approaches significance $(\mathrm{t}=-2.103 ; \mathrm{df}=17 ; \mathrm{p}=.051)$.

An ecologically valid driving simulator outcome variable, which addressed excursions over the center line or road excursions is referred to as Out of Lane. For both vehicles the Out of Lane count was triggered each time the vehicle traveled 1 foot over the lane marking on the left or right side. The scores for Out of Lane are shown in . The STISIM was not sensitive to detecting 
changes in this Out of Lane variable $(\mathrm{p}=.25)$. By contrast the MniSim detected a significant deterioration in the number of failures to remain in the driving lane $(\mathrm{t}=-2.533, \mathrm{df}=17 ; \mathrm{p}=.021)$.

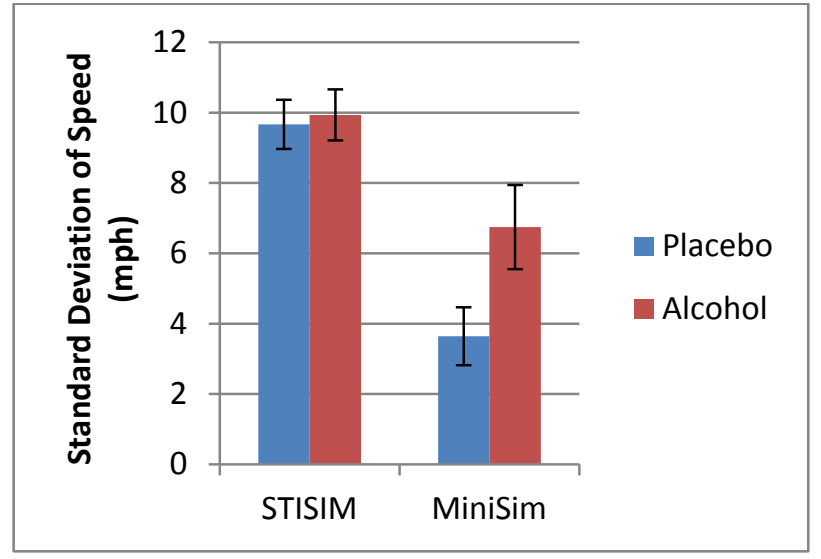

Figure 2. Variability in Speed

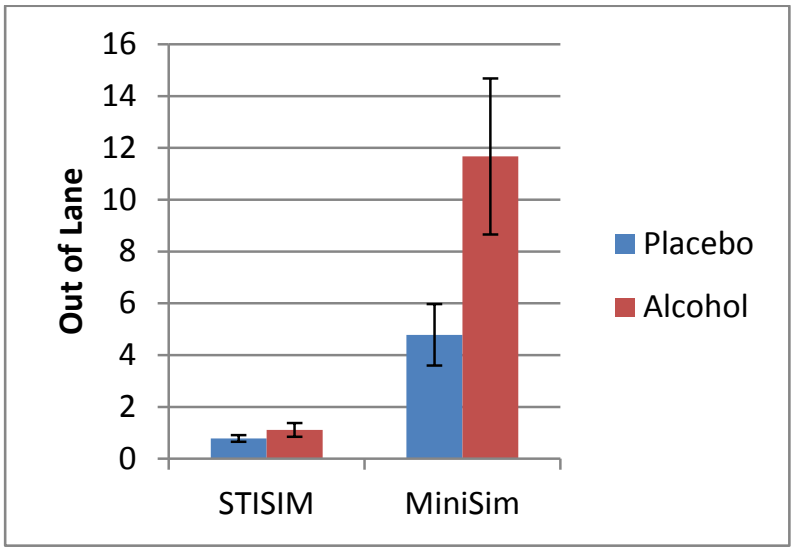

Figure 3. Frequency out of lane

A driving variable that has often been reported to be sensitive to the effects of alcohol, and which is related to a primary cause for alcohol-related crashes, is Excessive Cornering Speed. There was a very low count for Excessive Cornering for STISIM (i.e., $<1.0$ for both conditions) and the simulator did not show a difference for the alcohol condition $(\mathrm{p}=.317)$. By contrast, the MiniSim detected Excessive Cornering events; 6.5 under the Placebo condition and 8.2 under the Alcohol condition. The difference in treatment conditions showed a trend for significance ( $\mathrm{t}=-$ 1.67, $\mathrm{df}=17 ; \mathrm{p}=.113)$.

\section{Divided Attention}

Table 3 shows the Paired Sample T-Test results for the two simulators on the Divided Attention task. As can be seen, the p-values are lower for each measure on the MiniSim in relation to the STISIM. Differences between the means are shown in Figure 4.

Table 3. Summary of Results for Divided Attention Task

\begin{tabular}{|l|l|l|}
\hline DAT Variable & \multicolumn{1}{|c|}{ STI Simulator } & MiniSim Simulator \\
\hline Correct Response & $\mathrm{t}=1.458, \mathrm{df}=17, \mathrm{p}=.163$ & $\mathrm{t}=2.028, \mathrm{df}=17, \mathrm{p}=.058$ \\
\hline Omission Errors & $\mathrm{t}=-1.58, \mathrm{df}=17, \mathrm{p}=.163$ & $\mathrm{t}=-2.028, \mathrm{df}=17, \mathrm{p}=.058$ \\
\hline Commission Errors & $\mathrm{t}=-1.00, \mathrm{df}=17, \mathrm{p}=.331$ & $\mathrm{t}=-3.215, \mathrm{df}=17, \mathrm{p}=.005$ \\
\hline Reaction Time & $\mathrm{t}=-1.47, \mathrm{df}=17, \mathrm{p}=.166$ & $\mathrm{t}=-1.751, \mathrm{df}=17, \mathrm{p}=.098$ \\
\hline Accuracy & $\mathrm{t}=1.529, \mathrm{df}=17 ; \mathrm{p}=.15$ & $\mathrm{t}=3.039, \mathrm{df}=17, \mathrm{p}=.007$ \\
\hline
\end{tabular}




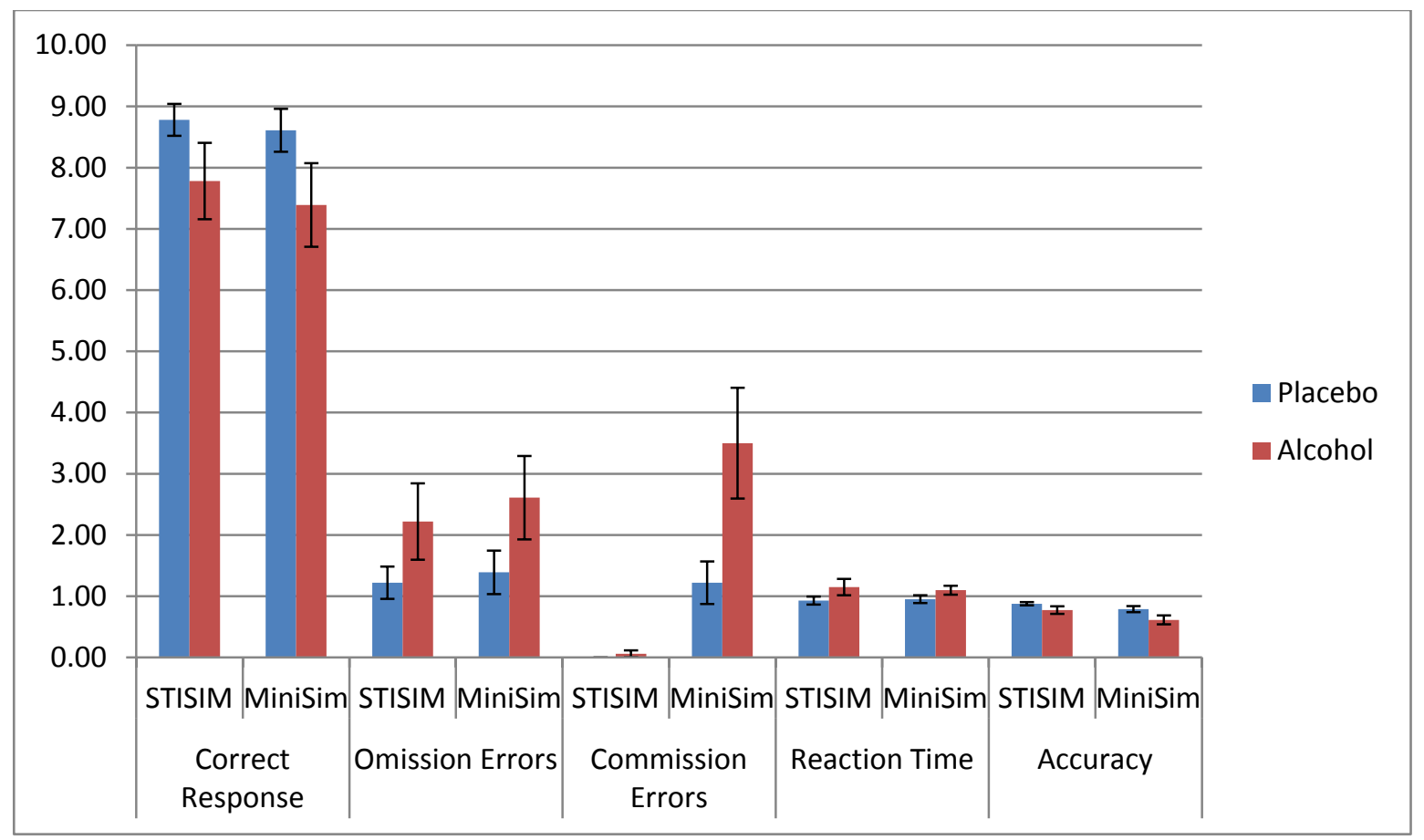

Figure 4. Divided Attention Measures

\section{CONCLUSION}

On driving variables and on divided attention variables the MiniSim simulator showed greater sensitivity to the impairing effects of alcohol (at doses below $0.10 \%$ BAC) than was found with the STI simulator. Table 4 shows the effect size (Cohen d) for the variables of interest in the study. The SDLP variable (lane position deviation) was sensitive to alcohol effects with both simulators; however, there was clearly greater sensitivity seen with the MiniSim simulator as represent by the greater effect size. For a number of driving and divided attention variables significant results were obtained with the MiniSim, whereas results for the STISIM failed to show a significant alcohol effect such as with standard deviation of speed.

Table 4. Effect Sizes

\begin{tabular}{|l|l|l|}
\hline Measure & STISIM & MiniSim \\
\hline Standard Deviation of Lane Position & 0.57 & 0.79 \\
\hline Standard Deviation of Speed & 0.09 & 0.73 \\
\hline Out of Lane & 0.38 & 0.73 \\
\hline Correct Response & 0.51 & 0.54 \\
\hline Omission Errors & 0.51 & 0.55 \\
\hline Commission Errors & 0.37 & 0.81 \\
\hline Reaction Time & 0.51 & 0.52 \\
\hline Accuracy & 0.53 & 0.69 \\
\hline
\end{tabular}

The greater sensitivity of the MiniSim compared to the STISIM may be due to a number of differences between the two simulators. These differences include: more realistic graphics leading to greater immersion into the simulated driving, faster data acquisition, and use of a more accurate vehicle dynamics model. The difference in the vehicle dynamics model and natural 
lane drift would be expected to be the largest determining factors. These would result in a greater effort being needed by drivers to maintain lateral and longitudinal control. Additionally, this greater workload would tax the drivers, particularly when impaired resulting in the greater effect associated with the divided attention task.

\section{ACKNOWLEDGEMENTS}

We would like to acknowledge Dr. Karim Abdel-Malek and the Center for Computer-Aided Design for their support of this research. Research was partially supported by an investigator initiated research grant received from Merck Sharp \& Dohme Corp.

\section{REFERENCES}

Cahalan, D., Cisin, I. \& Crossley, H. (1969). American drinking practices: A national study of drinking behavior and attitudes. New Brunswick, NJ: Rutgers Center of Alcohol Studies, Monograph No 6.

Iudice, A., Bonanni, E., Maestri, M., Nucciarone, B., Brotini, S., Manca, L., Iudice, G., \& Murri, L. (2002). Lormetazepam effects on daytime vigilance, psychomotor performance and simulated driving in young adult healthy volunteers. International Journal of Clinical Pharmacology and Therapeutics. 40(7): 304-309.

Kay, GG, Feldman N. (in press) Effects of armodafinil on simulated driving and self-report measures in obstructive sleep apnea patients prior to treatment with continuous positive airway pressure. Journal of Clinical Sleep Research.

Kay GG, Michaels MA, Pakull, B. (2009). Simulated driving changes in young adults with ADHD receiving mixed amphetamine salts extended release and atomoxetine. Journal of Attention Disorders. 12(4): 316-329.

Lee, J., Fiorentino, D., Reyes, M., Brown, T., Ahmad, O., Fell, J., Ward, N. \& Dufour, R. (2010). Assessing the Feasibility of Vehicle-Based Sensors to Detect Alcohol Impairment. Washington, DC: National Highway Traffic Safety Administration. DOT HS 811358.

Partinen, M., Hirvonen, K., Hublin, C., Halavaara, M., \& Hiltunen, H. (2003). Effects of aftermidnight intake of zolpidem and temazepam on driving ability in women with nonorganic insomnia. Sleep Medicine, 4(6): 553-561.

Saunders, J., Aasland, I., Babor, T., De La Fuente, J. \& Grand, M. (1993). Development of the Alchol Use Disorders Identification Test (AUDIT): WHO Collaborative Project on Early Detection of Persons with Harmful Alcohol Consuption - II. Addiction. 88: 791-804.

Thompson, I., Williams, G., Caldwell, B., Aldington, S., Dickson, S., Lucas, N., McDowall, J., Weatherall, M., Robinson, G. \& Beasley, R. (2010). Randomised double-blind, placebocontrolled trial of the effects of the 'party pills' BZP/TFMPP alone and in combination with alcohol. J Psychopharmacol, 1299-1308)

Veit, A. (2011). www.nads-sc.uiowa.edu/minisim. 